\title{
Theta burst repetitive transcranial magnetic stimulation attenuates somatosensory evoked potentials from the lower limb
}

\author{
Christopher M Zapallow', Michael J Asmussen ${ }^{1}$, David A E Bolton ${ }^{1}$, Kevin G H Lee ${ }^{1}$, Mark F Jacobs ${ }^{1}$ \\ and Aimee J Nelson ${ }^{1,2^{*}}$
}

\begin{abstract}
Background: Continuous theta burst stimulation (CTBS) is a form of repetitive transcranial magnetic stimulation which has been shown to alter cortical excitability in the upper limb representation of primary somatosensory cortex (SI). However, it is unknown whether cTBS modulates cortical excitability within the lower limb representation in SI. The present study investigates the effects of cTBS over the SI lower limb representation on cortical somatosensory evoked potentials (SEPS) and Hoffmann reflex (H-reflex) following tibial nerve stimulation at the knee. SEPs and H-reflex were recorded before and in four time blocks up to 30 minutes following CTBS targeting the lower limb representation within SI.

Results: Following CTBS, the P1-N1 first cortical potential was significantly decreased at 12-16 minutes. CTBS also suppressed the P2-N2 second cortical potential for up to 30 minutes following stimulation. The H-reflex remained statistically unchanged following CTBS although there was a modest suppression observed.

Conclusion: We conclude that CTBS decreases cortical excitability of the lower limb representation of SI as evidenced by suppressed SEP amplitude. The duration and magnitude of the CTBS after effects are similar to those observed in upper limb studies.
\end{abstract}

Keywords: Continuous theta burst stimulation, Somatosensory evoked potentials, Hoffmann reflex, Lower limb

\section{Background}

Decades of research in animal models have formulated principles that underlie plasticity within the primary somatosensory cortex (SI) [1]. In humans, plasticityinducing transcranial magnetic stimulation (TMS) protocols may be applied over SI to promote rehabilitation following injury [2], investigate somatic influences in motor control [3] and to understand the neural basis of touch perception [4]. To date, studies have focused primarily on altering excitability of upper limb representations within SI. However, investigating the effects of these same protocols on lower limb representations would provide insight into both the generalizability of these plasticity- inducing techniques to other body representations, and potentially lead to the development

\footnotetext{
* Correspondence: ajnelson@uwaterloo.ca

'Department of Kinesiology, University of Waterloo, Waterloo, Canada

${ }^{2}$ Department of Kinesiology, McMaster University, Hamilton, Canada
}

of new strategies to alter cortical activity in patients with sensory and/or motor impairments affecting the lower limb.

Continuous theta burst stimulation (cTBS) is a plasticity-inducing protocol that alters activity within SI such that somatosensory evoked potentials (SEPs) are decreased [5,6]. Specifically, cTBS applied over SI suppresses low frequency median nerve SEP components for up to 13 minutes [5] or leaves them unchanged [6]. High frequency oscillations are also modified by cTBS over SI such that early and late components are facilitated and suppressed, respectively, for up to 15 minutes following stimulation [6]. Of the plasticity-inducing protocols, cTBS offers the advantage of requiring short stimulation durations (i.e. 40 seconds) and relatively long-lasting effects [7]. Although the mechanisms of cTBS effects are not fully understood, cTBS appears to alter inhibitory and excitatory circuitry such that GABAergic $[8,9]$, 
glutamatergic $[10,11]$ and dopaminergic [12] systems are altered following stimulation. Studies in rat models reveal that TBS alters inhibitory neuronal circuitry within targeted cortex $[13,14]$. In support of these findings, cTBS increases GABA concentration within human cortex as measured using magnetic resonance spectroscopy [9]. However, NMDA receptor blockade eliminates the effects of cTBS thereby suggesting that glutamatergic systems are also influenced by cTBS $[10,11]$. Similarly, blockade of the dopamine D2 receptor eliminates the TBS effects [12].

To date, cTBS protocols have aimed to alter the excitability of upper limb representations within SI $[5,6]$ and M1 [5,7,15]. However, there exist distinct differences between hand and lower limb which may cause cTBS induced effects to differ between skin surfaces. Within SI of non-human primates, the hand representation exhibits a much larger cortical territory and higher cortical magnification factor than the lower limb [16]. The lower limb representation is intersected by the foot representation, resulting in two discrete foci dedicated to the lower limb, a trait not seen in the upper limb and hand $[16,17]$. Last, the glabrous skin of the hand has a propensity for inducing experience-dependent plasticity within SI $[18,19]$ whereas hairy skin, as seen in the lower limb, does not [20]. Further evidence is also found in humans such that a greater number of cortical synapses are involved in the processing of upper limb versus lower limb afferent input [21] thereby increasing the opportunity to modify afferent processing originating from the upper limb. Last, plasticity induced by the lower limb is not identical to that induced by other non-upper limb areas, such as the face. Following rTMS over the leg area, the motor output of ischemic-nerve-blocked upper limb was unchanged, whereas rTMS over the face resulted in long lasting decreases [22]. The aforementioned findings lead to the suggestion that the hand and upper limb may be targeted and modulated via cTBS protocols to a greater extent and with greater ease compared to the lower limb.

The goal of the present study was to investigate the effects of cTBS applied over the lower limb representation of SI on cortical excitability as measured with SEPs. The ability to modify cortical excitability within lower limb representations creates new opportunities for developing therapeutic strategies in clinical populations who demonstrate impairments in lower limb control. Further, it remains to be seen whether cTBS effects on upper limb SEPs may be generalized to lower limb representations. To address these questions SEPs were recorded before and for up to 30 minutes following cTBS over the lower limb representation within SI. Further, we obtained measurements of Hoffman reflexes (H-reflexes) in soleus muscle to identify whether cTBS induced effects occur at the cortical and/or spinal level. Based on the timeline of cTBS effects on early median nerve SEPs [5] it was hypothesized that a decrease in the first tibial nerve cortical potential would be seen up for up to 13 minutes following stimulation.

\section{Methods \\ Participants}

Nineteen healthy participants were tested (14 male, mean age $=25.1, \mathrm{SD}=5.24)$. Thirteen individuals participated in Experiment 1 (10 male, mean age $=25.2, \mathrm{SD}=5.0)$, six in control Experiment 2 (4 male, mean age $=20.5, \mathrm{SD}=$ 1.22), and three in control Experiment 3 (3 male, mean age $=25.7, \mathrm{SD}=3.06)$. Three individuals participated in both Experiments 1 and 3 ( 3 male, mean age $=25.7$, $\mathrm{SD}=3.06$ ). All participants were right handed, as determined by the Waterloo Handedness questionnaire, a derivative of the Edinburgh Handedness Inventory [23]. All participants wore $30 \mathrm{~dB}$ earplugs throughout all experimental procedures. All participants provided informed written consent prior to the experiment. This study conformed to the Declaration of Helsinki and was approved by the University of Waterloo.

\section{Electromyography (EMG)}

Muscle activity was recorded over the right soleus (SOL) and first dorsal interosseous (FDI) muscles using $9 \mathrm{~mm}$ $\mathrm{Ag}-\mathrm{AgCl}$ surface electrodes. For SOL, two electrodes were positioned approximately $2 \mathrm{~cm}$ apart over the muscle belly. For FDI, the active electrode was placed over the muscle belly while the reference electrode was placed over the metacarpophalangeal joint of the index finger. EMG was amplified 1000x and filtered from $20-2500 \mathrm{~Hz}$ using an Intronix Model 2024F isolated preamplifier (Intronix Technologies Corporation, Bolton, Canada) and acquired using Signal Software and a Cambridge Electronic device (Power 1401, Cambridge Electronic Design, Cambridge, $\mathrm{UK})$.

\section{Somatosensory evoked potentials}

The tibial nerve was stimulated (square wave pulse, 0.5 ms duration, $0.5 \mathrm{~Hz}$ ) at the popliteal fossa (Grass SD 9, Grass Technologies, West Warwick, USA) with the anode of the stimulating electrode placed approximately $2 \mathrm{~cm}$ proximal to the popliteal crease, and the cathode proximal to the anode, to elicit an H-reflex in SOL [24]. Nerve stimulation intensity was maintained at an intensity to evoke an $\mathrm{M}$ wave of $10 \%$ of the maximal $\mathrm{M}$ wave. The $10 \% \mathrm{M}$ wave intensity was monitored and maintained throughout the experiment.

SEPs were recorded from $\mathrm{CPz}$ located over the somatosensory vertex and referenced to $\mathrm{FPz}$ [25] with a clavicle ground. In 8 participants, we were able to additionally obtain a MRI to confirm the location of $\mathrm{CPz}$ over $\mathrm{SI}$ 
using Brainsight Neuronavigation Software (Rogue Research, Montreal, Canada), as shown in Figure 1A. MRI was collected with a 3 Tesla GE scanner (172 images) with 3DFSPGR-IR sequences using a $20 \mathrm{~cm}$ FOV (256x256). EEG recordings were amplified 10000x and filtered from 2-500 Hz (Intronix 2024F Isolated Preamplifier, Intronix Technologies Corporation, Bolton, Canada). Electrode impedances were maintained at $<5 \mathrm{k} \Omega$ throughout the experiment (UFI Checktrode, Model 1089 Mk III, UFI, Morro Bay, California, USA). SEPs were collected in epochs of 150 ms including a 30 ms pre-stimulus period. SEPs were averaged from 120 nerve stimuli delivered every 2 seconds. Previous studies examining tibial nerve evoked SEPs used 50 to 80 [26], $100[27]$ to $128[28,29]$ nerve stimuli. The frequency of stimulation was chosen to ensure $\mathrm{H}$-reflexes were not suppressed.

\section{Transcranial magnetic stimulation (TMS)}

TMS was applied using a MagPro stimulator (MCF-B65, Medtronic, Minneapolis, Minnesota, USA) using a $90 \mathrm{~mm}$ diameter figure-of-eight coil. The coil was positioned over left $\mathrm{M} 1$ at a $45^{\circ}$ angle to the mid-sagittal line to evoke motor evoked potentials (MEPs) in the right FDI. Once an optimal location for FDI MEPs was located, the resting motor threshold (RMT), defined as the lowest intensity required to evoke MEPs of at least $500 \mu \mathrm{V}$ in 5 of 10 consecutive trials while the muscle is at rest, was found. RMT was determined at the beginning of the experiment prior to nerve stimulation set-up. Continuous theta burst stimulation (cTBS) using 600 pulses [7] was applied over the location of the sensory vertex electrode $(\mathrm{CPz})$. We chose to use a cTBS intensity determined from RMT obtained from FDI based on a pilot study in which we noted that MEPs from the tibialis anterior and soleus muscles were inconsistent, frequently evoked activity in gluteal, hamstrings and quadriceps muscles, and required high intensities (i.e. 85-95\% of the MSO) using our figure-of-eight coil which created participant discomfort. Further, cTBS applied at these intensities may result in current spread, making inferences about results due to changes in SI difficult. To improve the opportunity to modify the excitability within the lower limb representation, we subsequently chose to increase the intensity

\section{A}

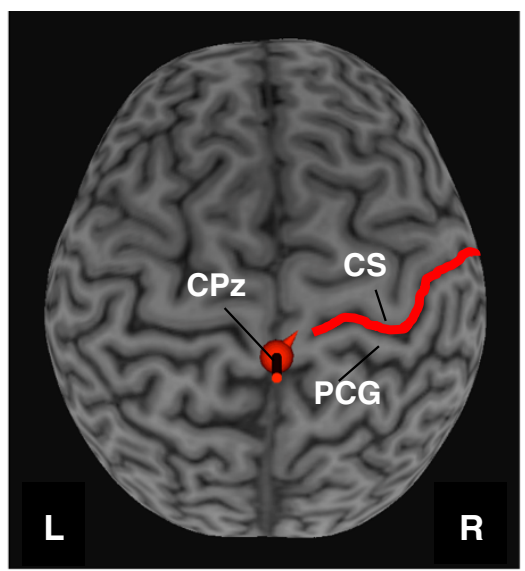

B

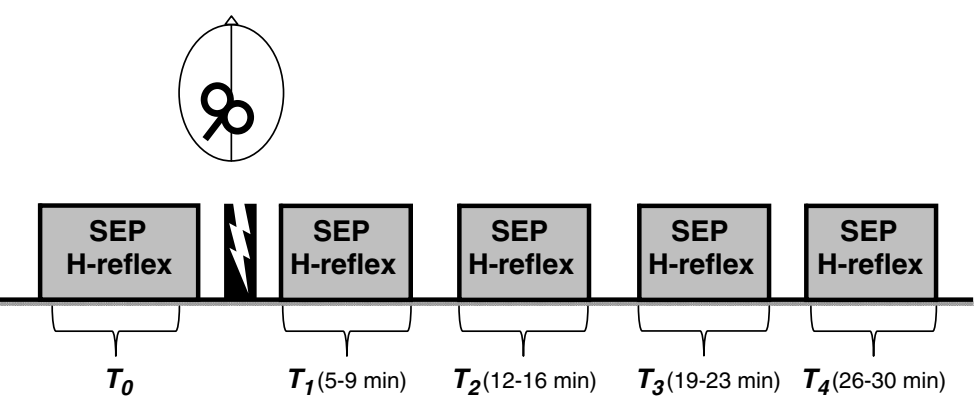

Figure $1 \mathrm{~A}$. Depiction of localization of cTBS target over CPz. CTBS was applied over the CPz location which was also the site for recording SEPs following tibial nerve stimulation. CS (central sulcus), PCG (postcentral gyrus). B. Experiment timeline. SEPs and H-reflexes were recorded before $\left(T_{0}\right)$ and at 5 to $9\left(T_{1}\right), 12$ to $16\left(T_{2}\right), 19$ to $23\left(T_{3}\right)$ and 26 to $30\left(T_{4}\right)$ minutes following SI cTBS. 
required for RMT and increase the cTBS intensity to $80 \%$ RMT, as opposed to the $80 \%$ active motor threshold value used in most upper limb studies. Further, we chose to use RMT rather than AMT to avoid metaplasticity effects of cTBS [30] which may contribute to the variable effects of cTBS observed elsewhere [31].

For cTBS, the coil handle was positioned to induce an initial current in the anterior to posterior direction. Prior to application of cTBS, the $\mathrm{CPz}$ electrode was removed from the scalp to ensure accurate application of the cTBS and following the completion of cTBS the $\mathrm{CPz}$ electrode was replaced at the original location on the scalp and impedances were checked to ensure proper reapplication similar to that performed elsewhere $[4,32]$. This procedure was performed to allow the cTBS coil to be placed directly upon the cranium though we recognize that it may also introduce some variability in the data.

\section{Experiment 1: Real cTBS over SI}

Thirteen subjects participated in Experiment 1. Measures of SEPs and H-reflexes were obtained immediately before cTBS $\left(T_{0}\right)$ and at four time blocks following stimulation: 5 to $9\left(T_{1}\right), 12$ to $16\left(T_{2}\right), 19$ to $23\left(T_{3}\right)$, and 26 to $30\left(T_{4}\right)$ minutes. The experiment timeline is shown in Figure 1B.

\section{Experiment 2: Sham cTBS over SI}

Six subjects participated. Experiment 2 was identical to Experiment 1 with the exception that Sham cTBS was applied over SI. For sham stimulation, the real cTBS protocol was delivered with the coil tilted perpendicular to the cranium and positioned over the $\mathrm{CPz}$ electrode position. In this orientation, the current flow is mainly directly towards outside of the cranium.

\section{Experiment 3: Extended duration real cTBS over SI}

Three subjects participated. Experiment 3 was identical to Experiment 1 with the exception that three additional time blocks were included to extend the recording window to 60 minutes following cessation of cTBS. These time blocks were 36 to $40\left(T_{5}\right), 46$ to $50\left(T_{6}\right)$, and 56 to $60\left(T_{7}\right)$ minutes following cTBS. Based on the findings of Experiment 1, this control experiment investigated whether P2-N2 changes returned to baseline by $60 \mathrm{~min}$ utes following cTBS.

\section{Data analysis}

Two one-way repeated measures ANOVA were performed, one analyzing peak-to-peak amplitude of SEP components and the H-reflex, and a second analyzing onset latency of SEP components and the H-reflex, both with within-subject factor TIME (5 levels: $T_{0} ; T_{1} ; T_{2} ; T_{3}$; $T_{4}$ ). Peak-to-peak SEP amplitudes were measured for the P1-N1 and P2-N2 tibial nerve cortical potentials by averaging 120 epochs during each time block. Trials displaying movement, blink, or noise artifacts were identified and rejected during off-line analysis. A priori hypotheses tested that $\mathrm{P} 1-\mathrm{N} 1$ would be suppressed at $T_{1}$ ( 5 to 9 minutes following cTBS) and $T_{2}$ (12 to 16 minutes following cTBS) as compared to $T_{0}$. Post-hoc analysis was performed using Tukey's tests.

\section{Results}

Experiment 1: Real cTBS over SI

All participants successfully completed the experiment. Data from one individual was removed due to movement related noise in the SEP recordings. Analyses were subsequently performed on data from the remaining 12 participants $(10$ male, mean age $=25.3, \mathrm{SD}=5.45)$. The group-averaged RMT and cTBS intensity (with standard deviation) were $55 \pm 8.4 \%$ and $44 \pm 6.8 \%$ of the maximum stimulator output (MSO), respectively. The latencies of the P1, N1, P2, N2 SEP components and H-reflex (with standard deviation) for each time block are shown in Table 1. With the exception of P2, the latencies of all components remained unchanged following cTBS (P1: $\mathrm{F}_{(4,44)}=0.71, \mathrm{p}=0.59, \mathrm{~N} 1: \mathrm{F}_{(4,44)}=0.68, \mathrm{p}=0.61, \mathrm{P} 2: \mathrm{F}_{(4,44)}=3.36$, $\mathrm{p}=0.017, \quad \mathrm{~N} 2: \mathrm{F}_{(4,44)}=0.99, \mathrm{p}=0.42, \quad$ H-reflex: $\mathrm{F}_{(4,44)}=0.0$, $\mathrm{p}=1.0$ ). Post hoc Tukey's test revealed that the latency of the P2 component was significantly altered at $T_{2}, T_{3}$, and $\mathrm{T}_{4}$ compared to $\mathrm{T}_{0}$. Therefore, the P2-N2 amplitude was analyzed two ways. Method one used the P2-N2 amplitude derived by the latency values obtained from the pre-cTBS block. Method two used the P2-N2 amplitude defined by the P2 latency from each time block separately (i.e. from $\left.\mathrm{T}_{0}, \mathrm{~T}_{1} \ldots.\right)$.

\section{Somatosensory Evoked Potentials}

Figure 2A displays the P1-N1 group-averaged peak-topeak amplitude with standard error bars. One-way ANOVA revealed no effect of TIME $\left(\mathrm{F}_{(4,44)}=1.53\right.$, $\mathrm{p}=0.21$ ). However, in support of the hypothesis, a priori comparison of pre-cTBS amplitude versus $\mathrm{T}_{2}$ revealed that $\mathrm{P} 1-\mathrm{N} 1$ is significantly suppressed from 12 to 16 minutes following cTBS (paired $t$-test, $\mathrm{p}=0.023$ ) and this effect was observed in 9 of 12 participants. P1-N1 suppression was also nearly significant at $T_{1}$ from 5 to 9 minutes following cTBS (paired $t$-test, $\mathrm{p}=0.03$ ).

Table 1 Group-average latency of SEP components and H-reflex (mean \pm SD)

\begin{tabular}{lccccc}
\hline & P1 & N1 & P2 & N2 & H-reflex \\
\hline T0 & $30(2.8)$ & $40(2.8)$ & $47(2.9)$ & $70(7.3)$ & $30(1.5)$ \\
\hline T1 & $31(3.6)$ & $41(4.3)$ & $48(4.3)$ & $67(7.4)$ & $30(1.7)$ \\
\hline T2 & $32(2.9)$ & $42(2.9)$ & $51(3.8)$ & $69(6.2)$ & $30(1.7)$ \\
\hline T3 & $31(3.2)$ & $42(3.7)$ & $49(3.7)$ & $69(6.7)$ & $30(1.7)$ \\
\hline T4 & $31(2.5)$ & $41(4.2)$ & $49(3.9)$ & $69(7.5)$ & $30(1.7)$ \\
\hline
\end{tabular}



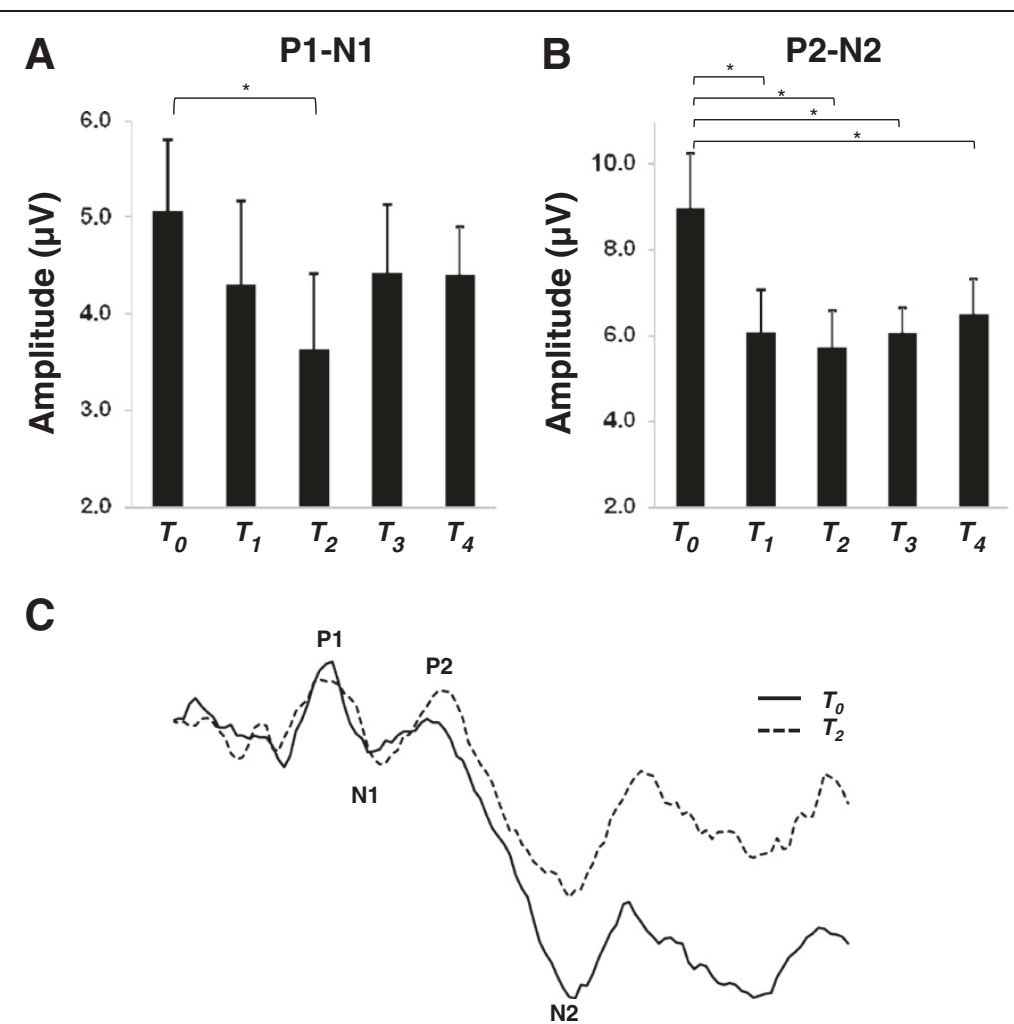

Figure 2 SEPs before and after cTBS. A. Group-averaged P1-N1 (with standard error) recorded before and at each time block following cTBS. B. Group-averaged P2-N2 (with standard error) recorded before and at each time block following CTBS. C. SEP trace from an individual participant depicting location of the P1, N1, P2 and N2 components at $T_{0}$ (before) and at time block $T_{2}$ (12 to 16 minutes) following cTBS. Asterisks indicate $p<0.05$.

Figure 2B displays the group-averaged P2-N2 amplitude with standard error bars. One-way ANOVA revealed a significant main effect of TIME for both analyses (Method one: $\mathrm{F}_{(4,44)}=9.81, \mathrm{p}=<0.0001$, Method two: $\mathrm{F}_{(4,44)}=3.72$, $\mathrm{p}=0.01$ ). Post-hoc Tukey's tests revealed that P2-N2 was significantly suppressed at each time block compared to pre-cTBS amplitude using Method one and at $T_{2}, T_{3}$ and $T_{4}$ using Method two.

\section{H-reflex}

Figure 3 displays group-averaged $\mathrm{H}$-reflex and $\mathrm{M}$ wave amplitudes with standard error for each time block. For the H-reflex, one-way ANOVA revealed no effect of TIME $\left(\mathrm{F}_{(4,44)}=1.77, \mathrm{p}=0.15\right)$ suggesting that $\mathrm{cTBS}$ over SI did not significantly alter spinal motor neuron excitability. Similarly, for the M wave, one-way ANOVA revealed no effect of TIME $\left(\mathrm{F}_{(4,44)}=0.56, \mathrm{p}=0.69\right)$ indicating that nerve stimulation intensity was maintained across time blocks.

\section{Experiment 2: Sham cTBS over SI}

All participants successfully completed the experiment. Data from one individual was removed due to excessively large potentials deemed to be statistical outliers in the pre-cTBS condition. Analyses were subsequently performed on data from the remaining 5 participants ( 3 male, mean age $=20.4, \mathrm{SD}=1.34$ ). The groupaveraged RMT and cTBS intensity (with standard deviation) were $51 \pm 8.9 \%$ and $41 \pm 7.3 \%$ of the MSO respectively. Figure 4 displays the group-averaged $\mathrm{M}$ wave, $\mathrm{H}$-reflex, P1-

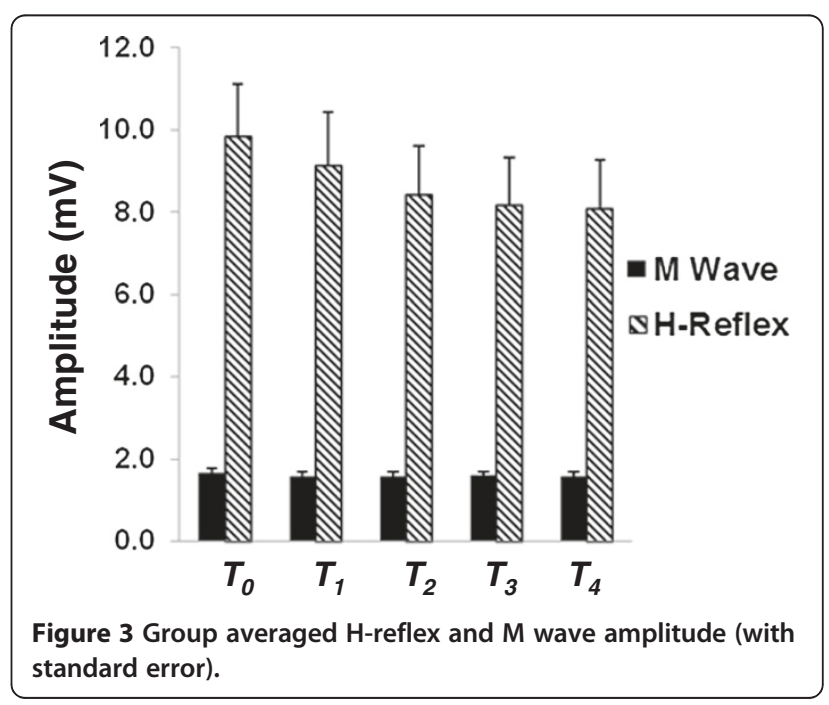




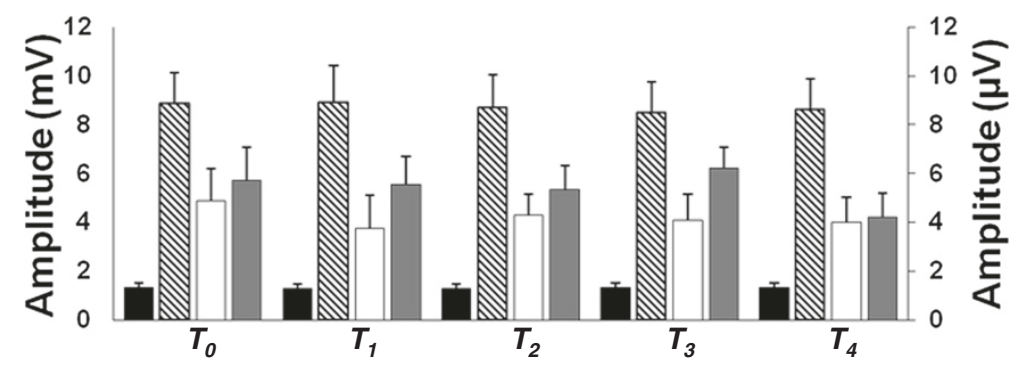

-M-wave $\mathbb{8}$ H-reflex $\square \mathrm{P} 1-\mathrm{N} 1 \quad \square \mathrm{P} 2-\mathrm{N} 2$

Figure 4 Sham cTBS over SI. Group averaged peak-to-peak amplitudes (with standard error) of SEPs and spinal reflexes before and at each time block following cTBS. No significance was found for any measure at any time point. The left ordinate reflects the $M$ wave and $\mathrm{H}$ reflex and the right ordinate scale reflects the SEPS.

N1, and P2-N2 peak-to-peak amplitudes (with standard error) for each time block. One-way ANOVA revealed no effect of TIME for $\mathrm{M}$ wave $\left(\mathrm{F}_{(4.16)}=0.48, \mathrm{p}=0.75\right)$, H-reflex $\left(\mathrm{F}_{(4.16)}=0.36, \mathrm{p}=0.83\right), \mathrm{P} 1-\mathrm{N} 1 \quad\left(\mathrm{~F}_{(4.16)}=1.52, \mathrm{p}=0.24\right)$, or $\mathrm{P} 2$ $\mathrm{N} 2\left(\mathrm{~F}_{(4.16)}=1.97, \mathrm{p}=0.15\right)$ suggesting that sham cTBS had no significant effect on either cortical or spinal excitability.

\section{Experiment 3: Extended duration real cTBS over SI}

All participants successfully completed the experiment. The group-averaged RMT and cTBS intensity (with standard deviation) were $56 \pm 15.1 \%$ and $45 \pm 12.1 \%$ of the MSO respectively. Figure 5 displays the groupaveraged $\mathrm{M}$ wave, $\mathrm{H}$-reflex, $\mathrm{P} 1-\mathrm{N} 1$, and $\mathrm{P} 2-\mathrm{N} 2$ peak-topeak amplitudes (with standard error) for each time block. The data suggest that the P2-N2 amplitude returns to baseline by 60 minutes following stimulation.

\section{Discussion}

In the present study we investigated the effects of cTBS over the SI representation of the lower limb on somatosensory evoked potentials and spinal reflexes originating from tibial nerve stimulation. Novel findings include suppression of the P1-N1 component from 12-16 minutes and reduced P2-N2 for up to 30 minutes following cTBS. CTBS did not significantly alter spinal H-reflexes.
These results indicate that cTBS exerts similar effects on lower and upper limb SEPs and that cTBS can be applied over SI to modulate the excitability of the lower limb representation.

Early cortical SEP potentials following median nerve stimulation are thought to originate from the arrival of the thalamo-cortical volley from the thalamus into area $3 \mathrm{~b}$ of SI [33] and a similar path is postulated for early cortical potentials following stimulation of the tibial nerve [34,35]. The P1 is generated by depolarization of the apical dendritic membrane of pyramidal neurons in the cortex, resulting in a relative electronegativity in reference to the cell soma and basal dendrites [36]. As this current propagates towards the soma and basal dendrites a dipole is formed with the negative pole being located at the apical dendrites (the "sink") and the positive pole being located at the basal dendrites (the "source") [36]. This dipole is recorded via EEG as a positivity as the electrode only records from the "visible" side of the dipole, with the negative dipole being occluded [36]. Conversely, the N1 results from the dipole generated by the same population of pyramidal cells with the "sink" being located at the basal dendrites, and the "source" being located at the apical dendrites [36]. The amplitude of these potentials reflects the magnitude of

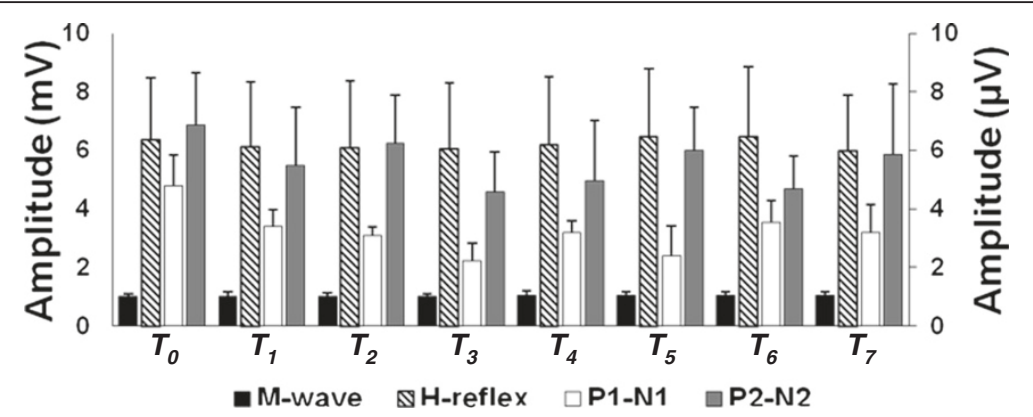

Figure 5 Extended duration real cTBS over SI. Group averaged peak-to-peak amplitudes (with standard error) of SEPs and spinal reflexes before and at each time block following CTBS. Three additional time blocks were added to extend the recording window to 60 minutes following cTBS. The left ordinate reflects the $\mathrm{M}$ wave and $\mathrm{H}$ reflex and the right ordinate scale reflects the SEPS. 
the summation of extracellular postsynaptic potentials created by the depolarization of the pyramidal neurons located at the generator site and the resultant dipole [36]. A suppression of the P1-N1 therefore reflects decreased depolarization of cortical pyramidal neurons and therefore decreased activity within this neuronal population.

The finding that tibial nerve P1-N1 SEPs are suppressed following cTBS closely matches results obtained from a study examining median nerve SEPs. Tibial nerve P1-N1 is suppressed for up to 16 minutes following cTBS but is not suppressed by 19 minutes. Median nerve P25-N33 potentials are suppressed for up to 13 minutes but are unchanged when tested at 20 minutes [5]. Further, the magnitude of cTBS induced suppression is similar for the lower and upper limb. CTBS decreases tibial nerve P1-N1 by $\sim 28 \%$ while median nerve P25$\mathrm{N} 33$ is reduced by $\sim 25 \%$ [5] or remains unchanged [6]. Comparison of the upper and lower limb data suggests that the time course and magnitude of changes induced by cTBS are similar for the first cortical potential for nerves of the upper and lower limb.

The generator of the tibial nerve derived P2-N2 is still largely unknown. However, somatosensory evoked fields following tibial nerve stimulation suggest that potentials occurring at approximately $50 \mathrm{~ms}$ arise from a generator located in area 1 of SI [35]. Further, the P2-N2 appears to originate from a similar large-diameter afferent source that may traverse a polysynaptic pathway before terminating within SI [37]. Similar to the P1-N1, P2-N2 decreased $\sim 36 \%$ with the maximal suppression occurring from 12 to 16 minutes following cTBS. However, unlike the earlier potential, P2-N2 was significantly decreased for up to 30 minutes with the suppression maintained at $\sim 30 \%$. The robust effects of cTBS on P2$\mathrm{N} 2$ compared to $\mathrm{P} 1-\mathrm{N} 1$ may relate to the spatial proximity between their respective generators and the position of cTBS delivery within SI. Area 1, where later occurring SEPs are thought to be generated, is located on the crown of the postcentral gyrus in close proximity to the focus of cTBS stimulation [38]. In comparison, area 3b, where early cortical potentials are generated, is located in the depths of the posterior bank of the central sulcus [38]. We also observed that the P2 latency increased at 12-16 minutes following cTBS which may also relate to the proximity of the cTBS delivery to the P2 generator. An alternative explanation is that cTBS alters activity in remote loci involved in the polysynaptic pathway through which the P2 travels before arriving in SI [37]. The latter explanation is plausible as cTBS is known to alter activity in remote loci [39].

The H-reflex remained statically unchanged both in latency and amplitude following cTBS. This agrees with previous work showing that cTBS over M1 did not alter
H-reflexes [7] or F-waves following Brodmann area 5 stimulation [40]. However, we note that the H-reflex did show modest suppression beginning at $\sim 12-16$ minutes following cTBS. Specifically, the H-reflex was suppressed by $\sim 15 \%$ from the pre-cTBS amplitude. This suggests that effects induced by cTBS over SI are mediated primarily via changes in the excitability of cortical neurons but may also include modest changes in the spinal motor neuron output. Intermittent TBS delivered for five consecutive days over M1 alters H-reflexes in patients with multiple sclerosis [41]. It may be that repeat sessions of cTBS over SI and/or M1 may exhibit changes in spinal motor neuron excitability.

\section{Conclusion}

The results of the present study indicate that cTBS may be used over SI to modify the excitability of the lower limb representation. These findings create new opportunities to investigate the utility of cTBS to modulate SI activity in clinical populations. Understanding the modulation of SI lower limb representations has importance in designing rehabilitation strategies for individuals with impaired gait or balance and/or altered somatosensory processing of the lower limb such as in stroke populations.

\section{Competing interests}

The authors declare that they have no competing interests.

\section{Authors' contributions}

CMZ conceived of the study, carried out the data collection, analyzed all data, and drafted the manuscript. DAEB assisted with the study design, data collection and editing of the manuscript. MJA, KGHL, and MFJ assisted with data collection, and aided in editing of the manuscript. AJN conceived of the study, assisted with data analysis and assisted with manuscript preparation. All authors read and approved the final manuscript.

\section{Acknowledgments}

We thank Natural Sciences and Engineering Research Council to AJN.

Received: 8 August 2012 Accepted: 23 October 2012

Published: 31 October 2012

\section{References}

1. Nudo RJ: Neural bases of recovery after brain injury. J Commun Disord 2011, 44:515-520.

2. Bashir S, Mizrahi I, Weaver K, Fregni F, Pascual-Leone A: Assessment and modulation of neural plasticity in rehabilitation with transcranial magnetic stimulation. PM R 2010, 2:S253-S268.

3. Tokimura H, Di Lazzaro V, Tokimura $Y$, Oliviero A, Profice $P$, Insola A, et al: Short latency inhibition of human hand motor cortex by somatosensory input from the hand. J Physiol 2000, 523(Pt 2):503-513.

4. Ragert $P$, Franzkowiak $S$, Schwenkreis $P$, Tegenthoff $M$, Dinse HR: Improvement of tactile perception and enhancement of cortical excitability through intermittent theta burst rTMS over human primary somatosensory cortex. Exp Brain Res 2008, 184:1-11.

5. Ishikawa S, Matsunaga K, Nakanishi R, Kawahira K, Murayama N, Tsuji S, et al: Effect of theta burst stimulation over the human sensorimotor cortex on motor and somatosensory evoked potentials. Clin Neurophysio/ 2007, 118:1033-1043.

6. Katayama T, Suppa A, Rothwell JC: Somatosensory evoked potentials and high frequency oscillations are differently modulated by theta burst stimulation over primary somatosensory cortex in humans. Clin Neurophysiol 2010, 121:2097-2103. 
7. Huang YZ, Edwards MJ, Rounis E, Bhatia KP, Rothwell JC: Theta burst stimulation of the human motor cortex. Neuron 2005, 45:201-206.

8. Cardenas-Morales L, Nowak DA, Kammer T, Wolf RC, Schonfeldt-Lecuona C: Mechanisms and applications of theta-burst rTMS on the human motor cortex. Brain Topogr 2010, 22:294-306

9. Stagg CJ, Wylezinska M, Matthews PM, Johansen-Berg $H$, Jezzard P, Rothwel

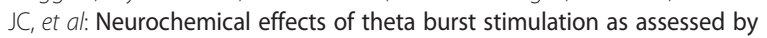
magnetic resonance spectroscopy. J Neurophysio/ 2009, 101:2872-2877.

10. Huang YZ, Chen RS, Rothwell JC, Wen HY: The after-effect of human theta burst stimulation is NMDA receptor dependent. Clin Neurophysiol 2007 118:1028-1032.

11. Teo JT, Swayne OB, Rothwell JC: Further evidence for NMDA-dependence of the after-effects of human theta burst stimulation. Clin Neurophysiol 2007, 118:1649-1651.

12. Monte-Silva K, Ruge D, Teo JT, Paulus W, Rothwell JC, Nitsche MA: D2 receptor block abolishes theta burst stimulation-induced neuroplasticity in the human motor cortex. Neuropsychopharmacology 2011, 36:2097-2102

13. Benali A, Trippe J, Weiler E, Mix A, Petrasch-Parwez E, Girzalsky W, et al: Theta-burst transcranial magnetic stimulation alters cortical inhibition. J Neurosci 2011, 31:1193-1203.

14. Trippe J, Mix A, Aydin-Abidin S, Funke K, Benali A: Theta burst and conventional low-frequency rTMS differentially affect GABAergic neurotransmission in the rat cortex. Exp Brain Res 2009, 199:411-421.

15. Suppa A, Ortu E, Zafar N, Deriu F, Paulus W, Berardelli A, et al: Theta burst stimulation induces after-effects on contralateral primary motor cortex excitability in humans. J Physiol 2008, 586:4489-4500.

16. Sur M, Nelson RJ, Kaas JH: Representations of the body surface in cortical areas $3 \mathrm{~b}$ and 1 of squirrel monkeys: comparisons with other primates. J Comp Neurol 1982, 211:177-192.

17. Nelson RJ, Sur M, Felleman DJ, Kaas JH: Representations of the body surface in postcentral parietal cortex of Macaca fascicularis. J Comp Neurol 1980, 192:611-643.

18. Xerri C, Merzenich MM, Jenkins W, Santucci S: Representational plasticity in cortical area $3 \mathrm{~b}$ paralleling tactual-motor skill acquisition in adult monkeys. Cereb Cortex 1999, 9:264-276.

19. Xerri C, Coq JO, Merzenich MM, Jenkins WM: Experience-induced plasticity of cutaneous maps in the primary somatosensory cortex of adult monkeys and rats. J Physiol Paris 1996, 90:277-287.

20. Coq JO, Xerri C: Environmental enrichment alters organizational features of the forepaw representation in the primary somatosensory cortex of adult rats. Exp Brain Res 1998, 121:191-204.

21. Kurusu K, Kitamura J: Long-latency reflexes in contracted hand and foot muscles and their relations to somatosensory evoked potentials and transcranial magnetic stimulation of the motor cortex. Clin Neurophysiol 1999, 110:2014-2019

22. Ziemann U, Wittenberg GF, Cohen LG: Stimulation-induced withinrepresentation and across-representation plasticity in human motor cortex. J Neurosci 2002, 22:5563-5571.

23. Oldfield RC: The assessment and analysis of handedness: the Edinburgh inventory. Neuropsychologia 1971, 9:97-113.

24. DeLisa J, Lee H, Baran E, Lai K, Spielholz N: Somatosensory Evoked Potentials. In Manual of Nerve Conduction Velocity and Clinical Neurophysiology. 3rd edition. Edited by. New York: Raven; 1994:195-274.

25. Seyal M, Emerson RG, Pedley TA: Spinal and early scalp-recorded components of the somatosensory evoked potential following stimulation of the posterior tibial nerve. Electroencephalogr Clin Neurophysiol 1983, 55:320-330.

26. Mcllroy WE, Bishop DC, Staines WR, Nelson AJ, Maki BE, Brooke JD: Modulation of afferent inflow during the control of balancing tasks using the lower limbs. Brain Res 2003, 961:73-80.

27. Terada K, Umeoka S, Baba K, Sakura Y, Usui N, Matsuda K, et al: Generators of tibial nerve somatosensory evoked potential: recorded from the mesial surface of the human brain using subdural electrodes. J Clin Neurophysiol 2009, 26:13-16.

28. Beric A, Prevec TS: Distribution of scalp somatosensory potentials evoked by stimulation of the tibial nerve in man. J Neurol Sci 1983, 59:205-214.

29. Vera $\mathrm{CL}$, Perot PL Jr, Fountain EL: Scalp recorded somatosensory evoked potentials to posterior tibial nerve stimulation in humans. Electroencephalogr Clin Neurophysiol 1983, 56:159-168.
30. Gentner R, Wankerl K, Reinsberger C, Zeller D, Classen J: Depression of human corticospinal excitability induced by magnetic theta-burst stimulation: evidence of rapid polarity-reversing metaplasticity. Cereb Cortex 2008, 18:2046-2053.

31. Hamada M, Murase N, Hasan A, Balaratnam M, Rothwell JC: The Role of Interneuron Networks in Driving Human Motor Cortical Plasticity. Cereb Cortex 2012, Epub ahead of print.

32. Premji A, Ziluk A, Nelson AJ: Bilateral somatosensory evoked potentials following intermittent theta-burst repetitive transcranial magnetic stimulation. BMC Neurosci 2010, 11:91.

33. Allison $\mathrm{T}$, McCarthy $\mathrm{G}$, Jones $\mathrm{SJ}$, Jones $\mathrm{SJ}$ : Potentials evoked in human and monkey cerebral cortex by stimulation of the median nerve. A review of scalp and intracranial recordings. Brain 1991, 114(Pt 6):2465-2503.

34. Kakigi $\mathrm{R}$, Jones $\mathrm{SJ}$ : Influence of concurrent tactile stimulation on somatosensory evoked potentials following posterior tibial nerve stimulation in man. Electroencephalogr Clin Neurophysiol 1986, 65:118-129.

35. Kakigi R, Koyama S, Hoshiyama M, Shimojo M, Kitamura $Y$, Watanabe S: Topography of somatosensory evoked magnetic fields following posterior tibial nerve stimulation. Electroencephalogr Clin Neurophysiol 1995, 95:127-134.

36. Gloor P: Neuronal generators and the problem of localization in electroencephalography: application of volume conductor theory to electroencephalography. J Clin Neurophysiol 1985, 2:327-354

37. Nelson AJ, Brooke JD, Mcllroy WE, Linklater CM, Staines WR: The afferent origin of the secondary somatosensory evoked potential from the lower limb in humans. Brain Res 2000, 887:432-435.

38. Nelson AJ, Chen R: Digit Somatotopy within Cortical Areas of the Postcentral Gyrus in Humans. Cereb Cortex 2008, 18:2341-2351.

39. Stefan K, Gentner R, Zeller D, Dang S, Classen J: Theta-burst stimulation: remote physiological and local behavioral after-effects. Neuroimage 2008, 40:265-274.

40. Premji A, Rai N, Nelson A: Area 5 influences excitability within the primary motor cortex in humans. PLoS One 2011, 6:e20023.

41. Mori F, Codeca C, Kusayanagi H, Monteleone F, Boffa L, Rimano A, et al: Effects of intermittent theta burst stimulation on spasticity in patients with multiple sclerosis. Eur J Neurol 2010, 17:295-300.

\section{doi:10.1186/1471-2202-13-133}

Cite this article as: Zapallow et al: Theta burst repetitive transcranial magnetic stimulation attenuates somatosensory evoked potentials from the lower limb. BMC Neuroscience 2012 13:133.

\section{Submit your next manuscript to BioMed Central and take full advantage of:}

- Convenient online submission

- Thorough peer review

- No space constraints or color figure charges

- Immediate publication on acceptance

- Inclusion in PubMed, CAS, Scopus and Google Scholar

- Research which is freely available for redistribution 